\title{
Enquêter en contexte de développement ou d'urgence : accès, risques, savoirs, restitutions
}

Compte rendu du colloque

APAD

\section{OpenEdition}

\section{Journals}

Édition électronique

URL : http://journals.openedition.org/anthropodev/484

DOI : 10.4000/anthropodev.484

ISSN : 2553-1719

Éditeur

APAD - Association pour l'anthropologie du changement social et du développement

\section{Édition imprimée}

Date de publication : 1 avril 2014

Pagination : 257-268

ISBN : 979-10-93476-00-1

ISSN : 2276-2019

Référence électronique

APAD, «Enquêter en contexte de développement ou d'urgence : accès, risques, savoirs, restitutions », Anthropologie \& développement [En ligne], 37-38-39 | 2014, mis en ligne le 01 décembre 2016, consulte le 23 septembre 2020. URL : http://journals.openedition.org/anthropodev/484; DOI : https://doi.org/ 10.4000/anthropodev.484

\section{(c) (i)}

La revue Anthropologie \& développement est mise à disposition selon les termes de la Licence Creative Commons Attribution 4.0 International. 


\section{Enquêter en contexte de développement ou d'urgence : accès, risques, savoirs, restitutions}

\section{Compte rendu du colloque}

Le $11^{\circ}$ colloque de I'APAD s'est déroulé à Montpellier, du 13 au 15 juin 2013. Le colloque se proposait d'approfondir les réflexions méthodologiques, épistémologiques et éthiques inhérentes à la relation d'enquête dans des situations humanitaires ou de développement caractérisées par des contextes de pauvreté, de détresse ou de violence et par l'intervention d'institutions, nationales ou internationales, qui cherchent à les gérer. Cette thématique prolonge celle qui avait été abordée lors du précédent colloque à Ouagadougou (Burkina Faso, 20-23 janvier 2010) sur les dimensions pratiques et éthiques de l'engagement de l'anthropologie dans le développement et le changement social ${ }^{1}$. En effet, l'affirmation d'un engagement de l'anthropologue dans les situations qu'il enquête l'interroge en tant que citoyen et sujet responsable (et pas seulement acteur) d'une réalité. Dans le contexte actuel de multiplication des conflits, de tensions sur la propriété intellectuelle des données d'enquête, ou sur le statut officiel de l'enquêteur, ces interrogations deviennent incontournables et stratégiques. Toutes les réflexions récentes sur le développement ${ }^{2}$ ont montré par ailleurs la profusion des acteurs (groupes stratégiques locaux, administration, ONG, projets, consultants, etc.), les difficultés croissantes d'accès au terrain ou à l'information, en particulier en situation de conflits, dans des camps de réfugiés mais aussi lorsque les institutions et acteurs politiques locaux produisent leur propre discours sur les réalités étudiées et s'érigent en "gardiens de la vérité ». Les rapports aux institutions de l'aide, qui financent parfois les études, y est plus que jamais ambivalent ; qui plus est, ces dernières sont devenues à leur tour objets de recherche, particu-

\footnotetext{
${ }^{1}$ Voir Bulletin APAD n ${ }^{\circledR 34-36}$ coordonné par S. Hagberg et F. Ouattara : Engaging Anthropology for Development and Social Change.

${ }^{2}$ Voir notamment Copans J., 2011, « Usages du développement : mémoires, politiques et sciences sociales ", Cahiers d'études africaines 2/2011 (N²02-203), p. 659-694.
} 
lièrement riches pour approfondir la réflexion éthique et épistémologique des anthropologues sur les situations de confrontation aux discours et connaissances produites par l'institution.

\section{Problématique du colloque}

\section{Les relations d'enquête, au cœur de la démarche anthropologique}

Les relations d'enquête, la façon de gérer ses relations avec les multiples acteurs rencontrés au cours d'une recherche, est au cœur de la démarche anthropologique, fondée sur un investissement personnel important dans la production de données et la recherche d'une " rigueur du qualitatif ». Les évolutions des objets de recherche ont à la fois déplacé et renouvelé cette question : le schéma classique de l'anthropologue étudiant les cultures des peuples lointains et s'installant dans un village sans forcément demander leur avis à ses habitants, est aujourd'hui périmé. Il a été remplacé par celui de chercheurs, prenant pour objet d'étude des enjeux placés au cœur de l'actualité, situés dans des rapports d'altérité variés avec les groupes sociaux qu'il étudie. Dans ce type de configurations, ils sont confrontés à une diversité d'acteurs institutionnels, politiques et médiatiques opérant ou enquêtant sur les mêmes terrains. La négociation de l'accès au terrain sur la longue durée, la légitimité du savoir anthropologique, la réflexivité des acteurs locaux sur leur propre situation, les attentes qu'ils placent dans le chercheur, les rapports aux institutions dominantes, sont devenues des questions désormais incontournables à la recherche ethnographique. Avec le tournant réflexif de la discipline, c'est aussi la prise de conscience de la relativité du savoir anthropologique et de son articulation à d'autres savoirs-experts (et politiques) qui s'est posée, et avec elle les questions de restitution, de mise en débat des résultats de recherche, et des usages qu'en font les acteurs étudiés.

\section{Tournant réflexif et nouveaux objets de l'anthropologie du développement}

Dans la dernière décennie, divers ouvrages et rencontres ont exploré ces mutations des politiques de l'enquête, en s'intéressant notamment aux 
implications de terrains dits "sensibles" ou encore à la question de "l'anthropologue en danger". Dans le champ du développement et de l'aide humanitaire, ce tournant réflexif a aussi été marqué par un renouveau de la réflexion, ancienne, sur les différents types de rapports entre recherche et action, et une exploration plus systématique de la portée heuristique des diverses postures possibles de l'anthropologue face aux institutions de l'aide et aux populations dites "vulnérables". Alors que l'anthropologie du développement s'est pendant longtemps polarisée entre critique radicale de l'entreprise développementaliste ou implication sans réflexivité dans l'action, des travaux novateurs adoptent aussi des postures plus nuancées, que ce soit sous forme d'un retour réflexif sur des expériences d'implication au sein de dispositifs projet ou en prenant pour objet d'études les logiques internes des institutions de l'aide, la production des savoirs-experts et des cadres d'action en leur sein ou encore le travail quotidien et les identités professionnelles des experts qui les constituent.

Ce renouveau réflexif a été d'autant plus indispensable que de nouveaux champs sont aujourd'hui explorés par les anthropologues du développement : les zones de conflits et de non droit, les camps de réfugiés et les situations dites d' "urgence ", les situations de violence quotidienne ou encore les institutions de l'aide vues de l'intérieur. Avec ces nouveaux objets, on mesure mieux encore à quel point l'anthropologue est en interactions permanentes avec une gamme élargie d'acteurs investis dans le champ de l'aide, à quel point il doit négocier sa place et ses relations, voire ses objets de recherche. A des degrés variés, la production anthropologique est modelée par ces interactions, et finalement, en partie coproduite par ces acteurs.

C'est en particulier le cas dans les expériences récentes de recherche pluridisciplinaires collaboratives, mais aussi les recherches menées dans le cadre d'expertises, qui tentent de plus en plus de structurer et d'institutionnaliser les relations entre chercheurs, institutions et enquêtés, ou d'explorer diverses formes de "partenariats épistémiques" avec les experts de l'aide. Ou dans les travaux récents de chercheurs ayant travaillé dans des institutions d'aide, qui ont ouvert des perspectives nouvelles sur la production des savoirs sur la pratique du développement. 
Les questions de confidentialité, d'anonymat, de risque que le chercheur fait prendre à ses informateurs, et d'engagement citoyen se posent aussi de façon nouvelle: obtenir le consentement des acteurs n'est pas suffisant, il faut aussi anticiper les conséquences de l'enquête afin de les protéger des répercussions potentiellement compromettantes voire dangereuses de leur participation.

\section{Pistes proposées pour les communications}

Le comité scientifique a rédigé un appel à communications soulignant l'importance qui serait donnée aux expériences de recherche collaborative avec des institutions d'aide et des praticiens et aux études analysant différentes dimensions du contexte de la recherche : la façon de négocier son accès au terrain; les difficultés d'accès à l'information ou les stratégies d'enclicage ou d'instrumentalisation; les risques que prend l'anthropologue et ceux qu'il fait prendre à ses différents interlocuteurs ; les questions de protection des informations et des informateurs et de propriété des données; la façon dont l'anthropologue influe sur son objet et dont, en retour, il est lui-même influencé par son positionnement particulier; la manière dont les relations d'enquête influent sur les stratégies d'écriture et de publication ou encore l'épineuse question de la confidentialité des données. L'appel à communication proposait par ailleurs trois axes de réflexion relatifs à des situations :

- les situations de violence quotidienne ou de pauvreté extrême, où la confrontation à la souffrance de l'autre se double parfois de prises de risques, pour l'anthropologue ou ses informateurs ;

- les situations d'urgence humanitaire, de guerre civile et de postconflit, où la violence, actuelle ou passée, se couple à l'intervention militaire et/ou humanitaire ;

- les situations d'enquête au sein des institutions d'aide, qu'elles soient centrées sur ces institutions elles-mêmes, sur leur personnel, ou sur leurs interventions, qu'elles soient réalisées de façon autonome ou à leur demande.

Comme pour les précédents colloques de I'APAD, l'appel à communications a sollicité des contributions émanant de chercheurs mais aussi d'acteurs du développement ou de l'humanitaire. Enfin, pour cette nou- 
velle édition, le Bureau de l'APAD a souligné sa volonté de ne pas limiter les études et réflexions aux situations d'aide au développement en Afrique et d'intégrer des contributions pouvant concerner d'autres continents.

\section{Organisation et déroulement}

Le colloque a été organisé grâce à l'investissement du Comité scientifique et du Comité d'organisation, en particulier un noyau de chercheurs montpelliérains des institutions partenaires (Cirad, IRD, Supagro). L'ensemble des financements a été géré par au CIRAD, par le service comptable et financier et le secrétariat de l'UR B\&SEF, Mme Annie Molina, Mme Pascale Hattot, Mme Marie-Aimée Schoukroun et Mme Isabelle Riquet. Les affiches et supports de communication ont été élaborés par Mme Valérie Rotival, responsable de la communication à l'IRD Montpellier. La préparation finale du colloque (gestion des inscriptions, organisation des panels, réalisation d'un livret papier des résumés des communications, préparation du dossier des participants - mallette, documents, clé USB des résumés -, organisation des hébergements, etc.) a bénéficié de l'appui d'un stagiaire, Félix Reimbolt, dont l'efficacité a été très largement appréciée. Nous les tous remercions vivement de leur implication.

Le colloque a été rendu possible grâce au soutien financier de diverses institutions : IRD (DIC-soutien aux colloques et Actions incitatives), la Direction de la recherche et de la Stratégie du Cirad, Supagro, les UMR GRED, ARTDEV et Innovation, I'UR 105 du Cirad, et la Région Languedoc Roussillon, et enfin le Département de la recherche de I'AFD, dont le généreux soutien a été déterminant. En particulier, nous avons pu financer la venue des deux conférenciers, d'une douzaine de chercheurs africains et apporter un appui à quelques étudiants européens. Les soutiens financiers reçus ont aussi permis d'assurer une traduction simultanée le premier jour, et d'organiser un cocktail de bienvenue le premier soir du colloque, au Moulin sur le site du CIRAD.

Le soutien financier dont a bénéficié ce colloque est d'autant plus crucial que I'APAD, association euro-africaine, a depuis sa fondation respecté la règle qu'elle s'était donnée de faciliter l'accès à ses colloques aux collègues africains (et aux étudiants), règle qui explique que le coût d'inscrip- 
tion au colloque ait été fixé à un très bas niveau. Contrairement à la majorité des événements scientifiques internationaux qui se déroulent actuellement, les colloques de I'APAD ne peuvent donc être organisés grâce aux montants des inscriptions. Ils dépendent bien plus que les autres de soutiens et d'engagements extérieurs.

Le colloque a été organisé sur deux sites, celui du Cirad (site de Lavalette) au sein du Campus Agropolis, et celui de Supagro (site de La Gaillarde), dans Montpellier même. Le déroulement a été organisé autour de deux conférences plénières introductives et de 5 temps de travail en sessions, chacune portant sur un grand thème. II a été ouvert par Patrick $\mathrm{Ca}-$ ron, Directeur général délégué à la science et à la stratégie, pour le Cirad, Laurent Vidal, directeur du Département Sociétés pour l'IRD), Alain Henry, Directeur du Département recherche de l'AFD, et Sten Hagberg, Président de l'APAD. Il a été clos par une table-ronde finale, et des interventions de Sten Hagberg et Isabelle Touzard, directrice adjointe de Supagro.

Les deux conférences plénières ont été données, en anglais par le $\mathrm{Pr}$ Tania Li (Université de Toronto) et en français par le Pr Loïc Wacquant (EHESS/Berkeley University). Elles visaient à mettre en perspective les thématiques du colloque et à ouvrir le champ de la réflexion. Intitulée "How do development institutions think : anthropological fieldwork inside development institutions ", la conférence du Pr Tania Li, fondée sur son expérience en Indonésie, questionnait la culture interne des institutions d'aide et les postures de l'anthropologue par rapport à elles. Fondée sur son expérience de terrain, la conférence de Loïc Wacquant, "Un sociologue français dans le ghetto noir américain : entrer, circuler, sortir " rappelait que les problèmes d'accès au terrain et de relations d'enquête ne sont pas spécifiques à l'anthropologie ni aux terrains du développement ou de l'urgence.

Un peu plus d'une centaine de résumés, dont un tiers en anglais, ont été reçus, en provenance de 25 pays. Environ 80 ont été acceptés. Nous avons eu des communications portant sur de nombreux pays, dont l'Iran, l'Inde, Haïti, le Kosovo, etc.

Les 20 sessions parallèles rassemblaient les communications autour de 5 thèmes distincts: Ethique et épistémologie de la relation d'enquête; Enquêter sur/ dans les marges et les conflits; Anthropologie sur/dans les 
institutions; Confrontation, négociation, coproduction des savoirs; Engagement et recherche collaborative. A chaque fois, une session a été organisée en langue anglaise, le comité d'organisation prévoyant que les participants au colloque n'auraient pas tous une bonne maîtrise de l'anglais et du français. Cette organisation qui tenait compte d'un critère linguistique essentiel, largement annoncée à l'avance dans les échanges avec les futurs participants, a permis que chacun s'exprime dans la langue où il se sent plus à l'aise, sachant que seules les deux conférences plénières du premier jour devaient bénéficier d'une traduction simultanée ${ }^{3}$ et qu'un résumé en anglais a pu être assuré lors de la table-ronde de clôture. La répartition en panels anglophones et francophones pour chacune des sessions a donc été très appréciée des participants.

Le programme a en outre compris un cocktail qui a été l'occasion de fêter les 20 ans de I'APAD avec quelques témoignages évoquant les grandes phases de l'histoire de l'association, et l'AG de l'APAD, qui a élu un nouveau bureau.

L'organisation des colloques de I'APAD prévoit aussi traditionnellement un temps dédié à l'Assemblée Générale de l'association. Les colloques sont en effet les seuls moments de rencontre physique (d'une partie) des membres. L'AG a été organisée le vendredi 14 juin entre $17 \mathrm{~h}$ et $18 \mathrm{~h} 30$, de manière à pouvoir réunir le plus grand nombre possible de participants, et sachant que ces derniers avaient aussi été invités dès leur accueil (ou dès leur première proposition de communication) à devenir membres de I'APAD. Cette AG est un moment clé de la vie de l'association puisqu'elle donne lieu au renouvellement des membres du Bureau, et à deux bilans (financier et moral) discutés collectivement.

Les différents échos et retour post colloque font état d'une grande satisfaction générale devant la qualité des débats et le mélange très apadien de débats approfondis dans une grande ouverture mutuelle et dans une ambiance détendue.

\footnotetext{
${ }^{3}$ A cause des grèves, la conférence de Loïc Wacquant a dû être reportée et n'a pu bénéficier de traduction simultanée. Il a lui-même assuré avec brio sa propre traduction.
} 


\section{Principaux points des contributions}

Nous retenons trois grandes entrées pour cette synthèse.

\section{Anthropologie dans les institutions, anthropologie des institutions}

Traditionnellement centrée sur l'interface locale entre projets de développement et sociétés locales, la socio-anthropologie du développement s'intéresse davantage aux institutions d'aide elles-mêmes, à leurs modes de prise de décision, à la trajectoire des experts, à la façon dont les cadres cognitifs sont produits et diffusés. En écho à un récent ouvrage en anglais ${ }^{4}$, plusieurs sessions ont été consacrées à ce thème, qui voulait balayer le continuum entre une anthropologie menant dans les institutions (Bliss, Burtscher pour MSF) des enquêtes ou du travail opérationnel avec les bénéficiaires des actions, et une anthropologie des institutions, où celles-ci sont objets de recherche (de l'intérieur ou de l'extérieur). De fait, alors que, dans sa conférence, Tania Li identifiait comme incompatibles les postures d'analyse critique et de contribution opérationnelle, les contributions ont largement exploré cette gamme de positions, insistant sur les postures hybrides (chercheur et praticien : Deligne, Mansion), les basculements (de praticien à chercheur : Enten, Scalettaris; ou de chercheuse à consultante temporaire ou évaluatrice : Gomez-Temesio, Ancey, Le Meur), les glissements successifs (Lavigne Delville) et ont analysé, dans une logique réflexive, leurs atouts, dilemmes et difficultés. Les institutions d'aide ont longtemps été considérées comme opaques, inaccessibles aux chercheurs, mais elles se laissent parfois assez aisément prendre comme objet de recherche (Fresia). L'autonomie laissée au chercheur, l'accès à certaines scènes, sont évidemment variées, comme dans n'importe quel terrain. La question de la confidentialité est parfois explicitement posée, d'autant plus que l'action de l'institution elle-même touche à des sujets confidentiels (Mora). Dès lors que l'institution a une forte emprise cognitive, et que l'on y a travaillé, en adhérent à cette culture, la " sortie du terrain " et la (re)construction d'un questionnement scientifique peut être difficile (Sca-

\footnotetext{
${ }^{4}$ Mosse D. ed., 2011, Adventures in Aidland. The Anthropology of Professionals in International Development, Coll. Studies in Public and Applied Anthropology Vol. 6, London, Berghahn.
} 
lettaris). Inversement, devenir " insider " d'une organisation après avoir été chercheur sur elle pose d'autres problèmes éthiques (Ngulube). Pour autant, ces postures hybrides ou intermédiaires se révèlent très productives scientifiquement, qu'elles permettent d'avoir accès à du matériau ou à des scènes normalement inaccessibles au chercheur, permette de rendre compte de la réflexivité des agents de développement (Deligne), de comprendre la fabrication des chiffres de l'aide alimentaire (Enten), d'analyser finement la culture interne d'une organisation (Hamani, Bourel), ses hiérarchies sociales (Segalini), la production des services (Bertho et Meda) ou la fabrique de la décision politique (Ousseini).

Ces communications montrent bien combien la question de l'anthropologie des institutions/dans les institutions ne peut être séparée d'un questionnement éthique et réflexif sur la position (les positions) du chercheur ou du praticien observateur, les relations qu'il noue avec ses collègues ou interlocuteurs de l'institution, et la tension entre incorporation de la culture de l'organisation (indispensable à la compréhension ethnographique) et distanciation analytique (indispensable à la construction scientifique). Les questions de la sortie du terrain, de la rupture ou renégociation du réseau relationnel $l^{5}$, du passage à l'écriture, sont ainsi cruciales.

\section{Enquêter en milieu difficile}

La question des "terrains sensibles " est inhérente à la recherche qualitative en sciences sociales. Plusieurs sessions en ont spécifiquement débattu dans le contexte spécifique de l'urgence ou du développement. Plusieurs textes discutent les conditions d'enquêtes en contexte de crise politique ou de violence : groupes armés en Côte d'Ivoire ou RDC (Chelpi den Hamer, Kouassi), crise politique au Mali (Hagberg). Dans ce contexte, c'est l'accès au terrain, aux acteurs de la violence qui est complexe, et peut engager des risques pour le chercheur. En Côte d'Ivoire, Kouassi et al. montrent comment la mobilisation de relations familiales est à la fois un atout et un piège. Hagberg discute la possibilité de poursuivre une re-

\footnotetext{
${ }^{5}$ Magnifiquement explicitées par Mosse quand il analyse la réception de son ouvrage « cultivating dévelopment " : Mosse D., 2006, "Anti-social anthropology? Objectivity, objection, and the ethnography of public policy and professional communities", Journal of the Royal Anthropological Institute, vol $12 \mathrm{n}^{\circ} 4$, pp. 935-956.
} 
cherche anthropologique lorsque l'accès au terrain devient impossible, en mobilisant à distance des informateurs. Seul un long terrain préalable donne la possibilité d'interpréter les informations produites indirectement. La difficulté du terrain peut aussi être inhérente à des régimes autoritaires, où le contrôle politique - sinon le risque physique - est fort (Sundberg pour le Rwanda, Saeidnia pour l'Iran, mais aussi Le Meur pour le Vietnam).

Une autre grande entrée traitée a concerné la question de la sorcellerie. Loin d'être une réminiscence du passé, l'interprétation sorcellaire se développe, de façon particulièrement criante en Afrique centrale, comme mode d'interprétation d'inégalités socio-économiques croissantes. La réussite des uns, la pauvreté et les échecs récurrents des autres, trouvent là un schéma d'intelligibilité. La violence (symbolique et/ou physique) liée à la sorcellerie pose des problèmes éthiques et méthodologiques délicats: pour l'anthropologue étranger, car la construire scientifiquement suppose d'échapper à l'ethnocentrisme qui la rejette tout comme aux cadres locaux d'interprétation qui la légitiment (Bouju) comme pour l'anthropologue autochtone (Ayimpam), dont l'intérêt pour ce sujet est vite suspect aux yeux de ses interlocuteurs, et qui doit conserver une neutralité axiologique vis-à-vis des présumés agresseurs comme des présumés victimes.

Un troisième axe fort a porté sur les situations d'extrême pauvreté (Arcens Somé) et les sujets tabous, et en particulier l'avortement (Ouattara, Ouedraogo). Ce dernier sujet, en particulier, est l'objet d'une réprobation sociale extrêmement vive en Afrique de l'Ouest, et d'une répression légale, alors même qu'il est largement pratiqué. Enquêter auprès de femmes qui ont avorté engage profondément la jeune chercheuse, dans ses émotions et ses propres convictions, qui doit remettre en cause des préjugés sociaux fortement ancrés, qui devient confidente et soutien de jeunes femmes en détresse (Ouedraogo). Abordant la question par le haut, F.Ouattara analyse finement les contradictions des responsables des politiques de santé et du personnel de santé, tiraillés entre leurs convictions personnelles et la détresse des femmes, et la façon dont les problèmes sanitaires postavortement sont pour certains une façon détournée de tenter de légitimer une réflexion de santé publique sur l'avortement. 


\section{Confrontation, négociation, coproduction des savoirs}

Le thème de la confrontation, la négociation et/ou la co-production des savoirs est au cœur du colloque où la relation d'enquête est revisitée dans ses contextes, les acteurs impliqués, leur diversité, leur hétérogénéité et les asymétries de pouvoir. Les communications ont montré combien ces interactions favorisent la réflexivité nécessaire à la production des savoirs et engendrent de nouveaux rapports au savoir où l'anthropologue renégocie sa posture et sa légitimité. Dans les situations de développement ou d'aide humanitaire la construction de la relation d'enquête y est interrogée (Bertho et Medah) dans sa capacité à engendrer une réflexivité des pratiques professionnelles. La construction de la relation d'enquête s'inscrit dans une production de confiance qui engendre des connaissances et réciproquement (Pesche). On comprend combien la réflexivité contient un pouvoir heuristique fort quand le contexte oblige à d'autres formes discursives. Elle participe alors à l'interprétation des silences et des secrets, des gestes muets et des mots entre les lignes des acteurs qui n'ont pas droit à la parole, et notamment des femmes marginalisées dans des contextes "dangereux, fragmentés, masculins et politisés " des interventions de I'aide et du développement (Grange Omokaro et Grabska). Cette réflexivité " réinterroge " également les postures de l'anthropologue "à la faveur d'une nouvelle rhétorique du développement » (Maizi- Moity)

La diversité des acteurs institutionnels, politiques et médiatiques reconfigure les relations entre chercheurs et acteurs du développement et de l'aide humanitaire. Les postures réciproques des anthropologues et des professionnels du développement ou de l'aide humanitaire se confrontent sur le terrain de la rhétorique comme sur celui de la pratique et donnent lieu à d'autres formes d'analyses plus fines et nuancées des conditions de la pratique. Dépassant la posture critique des uns, la " rigidité des schémas d'analyse " (Caremel) des autres, la posture de médiation dans la recherche anthropologique permet de faire émerger des lectures moins négatives de l'évaluation de projet en tentant de réintégrer la parole des différents acteurs dans les processus d'évaluation (Leclercq), voire " d'infléchir des positions normatives et juridiques pour y induire des perspectives processuelles et pragmatiques " (Imorou). Posture processuelle mais où le risque d'institutionnalisation de la médiation n'est pas nécessairement contreproductif sur la réflexivité (Bertho et Medah). La 
thématique de la confrontation, négociation et co-production des savoirs inclut les questions d'éthique et d'épistémologie de la relation d'enquête, autre thème du colloque. Ainsi, les rapports aux institutions dominantes de l'aide et du développement, aux acteurs politiques, aux représentants de groupes sociaux organisés qui produisent leur propre discours, rendent plus délicate la négociation de l'accès au terrain sur la longue durée et questionnent légitimité du savoir anthropologique. Mais la confrontation des savoirs peut aussi se cristalliser dans des productions épistémiques exclusives, voire des fondements ontologiques spécifiques comme le montre le cas de certaines communautés amérindiennes au Canada (Boutinot et Martin).

\section{Projets de valorisation scientifique}

Un premier bilan scientifique a permis d'identifier les pistes éditoriales pour valoriser les communications du colloque. Le projet initial portait sur un numéro spécial du Bulletin de l'APAD. La richesse des communications, sur différents thèmes, amène à s'engager dans plusieurs projets complémentaires, qui seront affinés après analyse approfondie des textes par les porteurs de ces différents projets :

- Un ouvrage "Anthropologie dans/des institutions". Editeurs scientifiques: Marion Fresia et Philippe Lavigne Delville, à proposer à la série APAD/Karthala ;

- Un numéro double ou triple du bulletin de l'APAD ( $\left.{ }^{\circ} 39-40\right)$ « Enquêter en terrains difficiles ». Editeurs scientifiques: Sylvie Ayimpam et Jacky Bouju. Trois sous-thèmes identifiés: Crises et violences; Accès au terrain et relation d'enquête dans contextes difficiles/contrôlés; Santé et sujets tabous (éventuellement couplé à un $\mathrm{n}^{\circ}$ de revue chez un autre éditeur pour un des thèmes) ;

- Un numéro de revue "Confrontation/coproduction de savoirs". Editeurs: Laurence Boutinot et Pascale Maizi-Moity. A proposer par exemple à la Revue d'Anthropologie des Connaissances. 